\title{
Sound synchronization of bubble trains in a viscous fluid: Experiment and modeling
}

\author{
Felipe Augusto Cardoso Pereira, ${ }^{1}$ Murilo da Silva Baptista, ${ }^{2}$ and José Carlos Sartorelli ${ }^{1}$ \\ ${ }^{1}$ Universidade de São Paulo, Instituto de Física, Caixa Postal 66318, 05315-970, São Paulo, Brazil \\ ${ }^{2}$ Institute for Complex System and Mathematical Biology, SUPA, University of Aberdeen, Aberdeen AB24 3UE, United Kingdom
}

(Received 2 June 2014; published 2 October 2014)

\begin{abstract}
We investigate the dynamics of formation of air bubbles expelled from a nozzle immersed in a viscous fluid under the influence of sound waves. We have obtained bifurcation diagrams by measuring the time between successive bubbles, having the air flow $(Q)$ as a parameter control for many values of the sound wave amplitude $(A)$, the height $(H)$ of the solution above the top of the nozzle, and three values of the sound frequency $\left(f_{s}\right)$. Our parameter spaces $(Q, A)$ revealed a scenario for the onset of synchronization dominated by Arnold tongues (frequency locking) which gives place to chaotic phase synchronization for sufficiently large $A$. The experimental results were accurately reproduced by numerical simulations of a model combining a simple bubble growth model for the bubble train and a coupling term with the sound wave added to the equilibrium pressure.
\end{abstract}

DOI: 10.1103/PhysRevE.90.042902

PACS number(s): 05.45.Xt, 05.45.Pq, 47.55.db

\section{INTRODUCTION}

Synchronization is ubiquitous in nature [1]. It has been observed in biological processes [2], such as ecological systems [3], postural control [4], and cardiorespiratory system in humans [5], coupled neurons [6,7] and neuron networks [8]. In complex networks, synchronization may appear as a combined effect of the dynamics of the nodes, their interactions, and the topological connecting structure of the network $[9,10]$.

Gas-liquid reactors have usually multiorifices or nozzles in the same plenum, where air bubbles interact in a complex way producing synchronous behaviors of the bubbling [11-14]. In Ref. [15], Snabre and Magnifotcham studied the effect of adding an independent bubble stream aside a previously ongoing one. They showed that the ongoing bubble stream rising velocity is reduced as the airflow of the added nozzle increases, and that the bubble streams attract each other, causing a small deviation of the bubble paths from the vertical. It was shown that synchronization depends on the distance between the orifices [12]. Synchronization and also bifurcations were shown in Ref. [14] to depend on the values of the airflow rates for the two orifices and the altitude of the liquid column [14]. This experimental observation was shown to be a direct consequence of the fact that the coupling between the two bubble trains is mediated by a change in the equilibrium pressure of the bubble proportional to the velocities of the bubbles in each train [16]. In Ref. [13], two synchronized modes were found. One for low and another for high airflow regimes, with nonperiodic behavior (intermittence) for moderate regimes.

Synchronization can also be the result of an external force being applied into a network or a self-sustained oscillator. Periodic perturbations applied to chaotic arrays [17] induce phase synchrony between the nodes, creating a physical media that allows information from the stimulus to propagate. That provides a simple explanation for how information can be transmitted in networks. Perturbing a nonlinear system by a periodic resonant signal has since long being a good strategy to increase the dimension of a system to study its bifurcations induced by resonances [18], such as a torus breakdown $[19,20]$, Arnold tongues [21,24], or chaotic phase synchronization [25,27]. It is also a simple strategy to study how a nonlinear system responds to complex periodic perturbations [28], or how it adapts its behavior having in mind potential technological applications where chaos needs to be suppressed [29-31] or induced [30,32].

In a previous experiment, see Ref. [33,34], about the dynamics of bubble formation under the effect of a periodic external signal, it has been shown that the amplitude of the sound waves was predominant in the appearance of limit cycles, flip bifurcation, chaos, and 1:1 frequency locking, when the frequency of the wave is equal to the inverse of the average period of the bubbling [33,34]. In Ref. [35], a methodological model based on two coupled circle maps could describe surprisingly well the behaviors experimentally obtained. This result showed evidences that this system could be described by a low-dimensional model. A more realistic still low-dimensional model based on an integrate-and-fire dynamics with a sinusoidal baseline was shown in Ref. [36] to reproduce remarkably well the long-time behavior of the instant of the bubble detachment.

Our interest in this work is to report the scenario for the onset of synchronization in the bubble formation under the influence of a periodic external signal, and propose a mathematical model for it. We will focus our attention in two phenomena: frequency locking and phase synchronization. Our parameters of interest are the airflow $Q$, the sound wave amplitude $A$, and the fluid height $H$ above the top nozzle. For a weak input signal (low $A$ ) we observe (periodic) frequency locking, a phenomenon characterized by a bubbling frequency that is a submultiple of the sound frequency [34-36]. For sufficiently strong input signal we observe (chaotic) phase synchronization, when the difference between the phases of the air bubbling and the external perturbation is bounded. This scenario is similar to the one reported in Ref. [37] for a self-excited oscillator with a double-well potential perturbed by a periodic signal. The action of the sound wave in the bubbling formation was modeled by introducing, in the realistic gas-liquid model proposed in Ref. [38], an equilibrium pressure [16] that is modulated by a cosine wave of amplitude $A$. Numerical results from this model have reproduced remarkably well all the main results observed experimentally. 


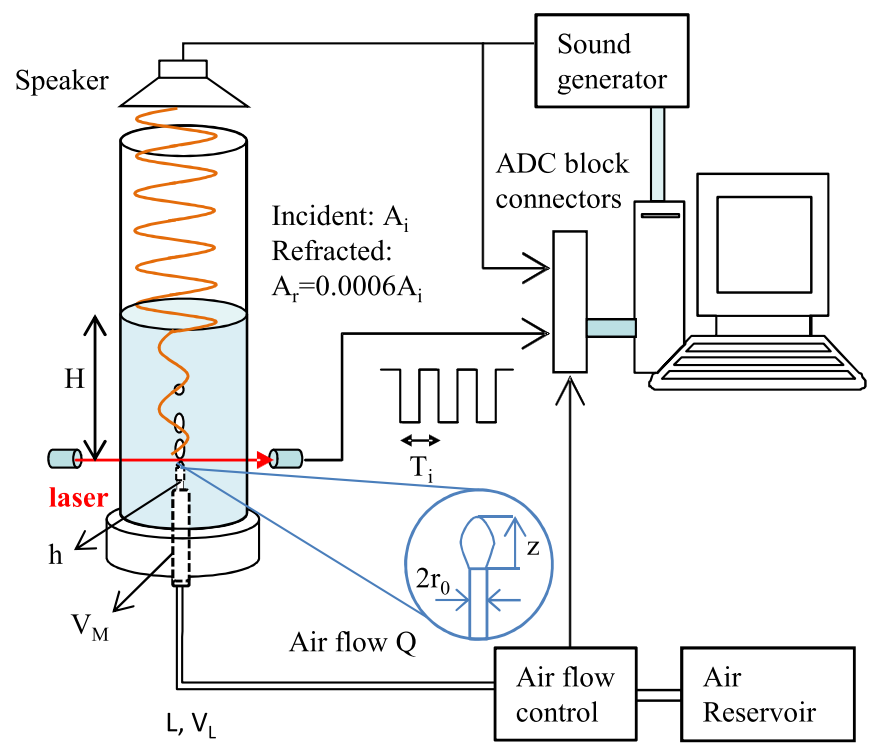

FIG. 1. (Color online) Schematic drawing of the experimental apparatus. The geometrical parameters are: Nozzle length $h=1.0$ $\mathrm{cm}$ and radius $r_{0}=1.1 \mathrm{~mm} ; V_{M}=10 \mathrm{ml}$ is the syringe chamber volume; $L=30 \mathrm{~cm}$ and $V_{L}=4.2 \mathrm{ml}$, respectively, are the length and the volume of the hose that connects the air flow control to the chamber. The zoomed view shows details of bubble profile. $H$ represents the viscous fluid height above the top of the nozzle and for $H=4,8.5,13 \mathrm{~cm}$ we have measured the time delay between successive bubbles having the airflow $Q$ as a parameter control in forward/backward sweepings.

\section{EXPERIMENTAL APPARATUS AND TIME-SERIES MEASUREMENTS}

In Fig. 1 is shown the diagram of the experimental apparatus. It consists of an acrylic transparent cylindrical container of $10 \mathrm{~cm}$ diameter which is partially filled with a glycerol and water solution (75\%-25\%), of density $\rho=1.21$ $\mathrm{g} / \mathrm{ml}$, kinematic viscosity $v=0.30 \mathrm{~cm}^{2} / \mathrm{s}$, and interface surface tension $\sigma=67 \mathrm{dyn} / \mathrm{cm}$. The bubbles are generated at $4.5 \mathrm{~cm}$ above the bottom of the solution container by injecting air through a nozzle, which is the tip of a hypodermic syringe, without needle, with a channel of length $10 \mathrm{~mm}$ and internal radius $1.1 \mathrm{~mm}$. The nozzle is connected to an air injecting system, with its airflow rate $Q$ controlled by a proportional solenoid valve driven by a PID controller [33-35,39] and measured with a flow meter in $\mathrm{ml} / \mathrm{min}$.

The time series of time intervals $T_{n}$ (in seconds) between successive bubbles are obtained by detecting in a resistor the beginning of the $5 \mathrm{~V}$ voltage pulses induced by the scattering of a laser beam (placed a little above the nozzle), focused in a photodiode which is in series with the resistor.

The mean time bubble formation and bubbling frequency (in bubbles/s) are respectively defined by

$$
T_{b}=\frac{1}{N} \sum_{n=1}^{N} T_{n}, \quad f_{b}=\frac{1}{T_{b}},
$$

where $N>100$ bubbles. We applied a sound wave with frequency $f_{s}$ tuned above the air column. The boundary conditions on the air-fluid interface shows that a small fraction of the incident wave is refracted to the solution, that is, $A_{r} \approx 0.0006 A_{i}$ (see Ref. [36]), still carrying enough energy to interfere significantly with the air bubble dynamics. We have constructed parameter spaces $(Q, A)$, in which the two parameter controls are the air flow $Q$ and the sound wave amplitude $A$, measured at the generator output, in arbitrary units $\left(A_{i} \propto A\right)$. For fixed values of $A$ we obtained time series $T_{n}$ by varying the air flow $Q$ in steps of $2 \mathrm{ml} / \mathrm{min}$. The time series of $T_{n}$ and the values of $Q$ were collected by a data acquisition system composed by an analog to digital converter (ADCs) of 16 bits at $250 \mathrm{ksamples} / \mathrm{s}$ measuring the signals simultaneously.

\section{EXPERIMENTAL RESULTS AND DISCUSSION}

\section{A. Frequency locking}

When bubbling is periodic we have that $T_{n+P}=T_{n}$, where $P \in \mathbb{N}$ is the discrete period, which is equal to the number of bubbles within one cycle. Additionally, $T_{i+2}=T_{i}$. The bubbling for period 1 is characterized by a constant value of the $T_{n}$ (so, $T_{i+1}=T_{i}$ ). Period 2 is characterized by a shorter time-interval $T_{i}$ followed by a longer time-interval $T_{i+1}$. An illustration of the bubbling behavior can be seen in Fig. 2. See Supplemental Material for a video showing the bubbling behavior for period 1 and period 2 [40]. The time of the complete cycle, that is, the period $T_{c c}$ in unit of time is given by

$$
T_{c c}=\sum_{i=1}^{P} T_{i} \approx P T_{b} .
$$

(a)

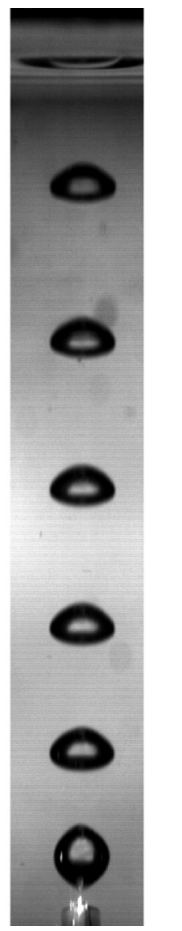

(b)

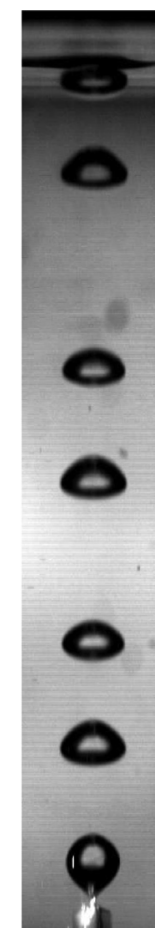

FIG. 2. Snapshots of bubbling for $Q=126 \mathrm{ml} / \mathrm{min}$. In (a) period $P=1$ without sound and in (b) of period $P=2$ for $A=5$. See Supplemental Material for a video showing changing in the bubbling behavior from period 1 to period 2 [40]. 
Frequency locking between the sound wave and periodic bubbling happens when the ratio between their frequencies (in $\mathrm{Hz}$ ) is a rational number:

$$
\frac{f_{c c}}{f_{s}}=\frac{T_{s}}{T_{c c}}=\frac{T_{s}}{P T_{b}}=\frac{N_{1}}{N_{2}},
$$

where $f_{c c}=1 / T_{c c}$ and $f_{s}$ is the frequency of the sound wave. Using that $f_{b}=\frac{1}{T_{b}}$ from Eqs. (1), and since $P, N_{1}, N_{2} \in \mathbb{N}$, we also must have that

$$
\frac{f_{b}}{f_{s}}=\frac{p}{q},
$$

$p=P N_{1}, q=N_{2} \in \mathbb{N}$. In practice, to verify frequency locking, we consider that Eq. (4) is valid if

$$
\left|\frac{f_{b}}{f_{s}}-\frac{p}{q}\right| \leqslant \epsilon
$$

where $\epsilon$ is of the order of $\epsilon \approx 2 \times 10^{-3}$, a value determined by observing how much $\left|\frac{f_{b}}{f_{s}}-\frac{p}{q}\right|$ is different from zero, in situations that the bifurcation diagrams of Figs. 3 and 4 indicate the existence of a periodic behavior.

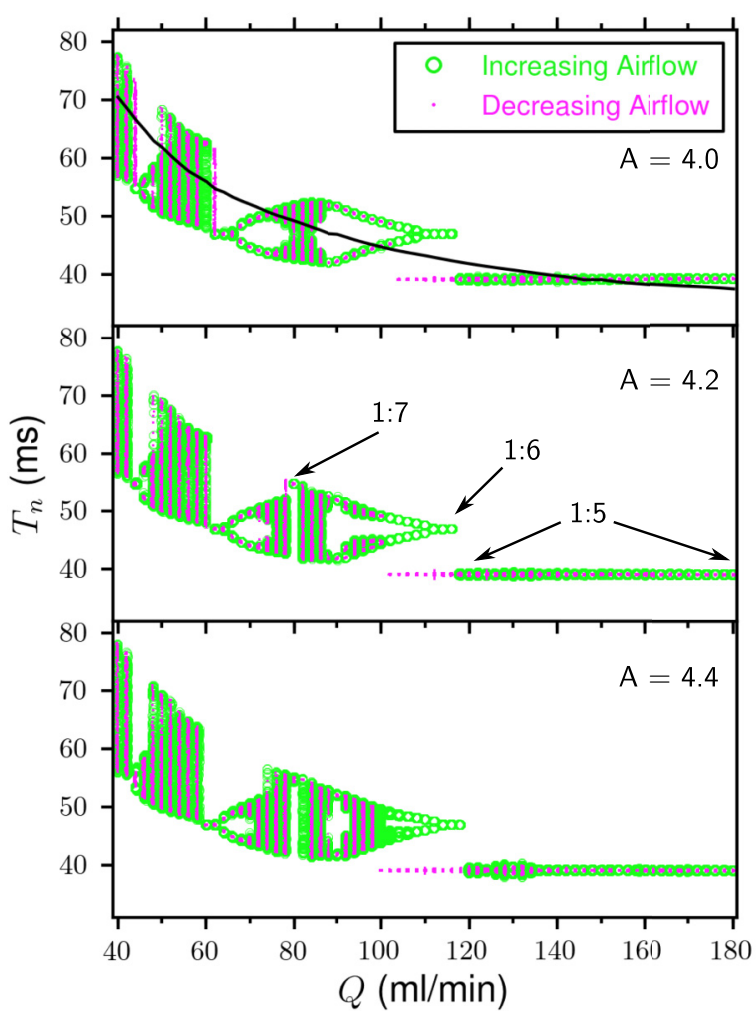

FIG. 3. (Color online) Experimental bifurcation diagrams $T_{n}$ vs $Q$, for $f_{s}=128 \mathrm{~Hz}$ and $H=8.5 \mathrm{~cm}$, obtained by increasing the air flow, in empty light gray circles (green) and decreasing the airflow $Q$, in full dark gray points (magenta) for $A=4.0$ (top panel), $A=4.2$ (middle panel), and $A=4.4$ (bottom panel). The black full line in the top panel represents the bifurcation diagram of a period- 1 orbit for no sound amplitude $(A=0)$. Frequency lockings $p: q=f_{b}: f_{s}$ are pointed out, $25.4 / 128=1: 5,21.33 / 128=1: 6$, and $18.28 / 128=$ $1: 7$.

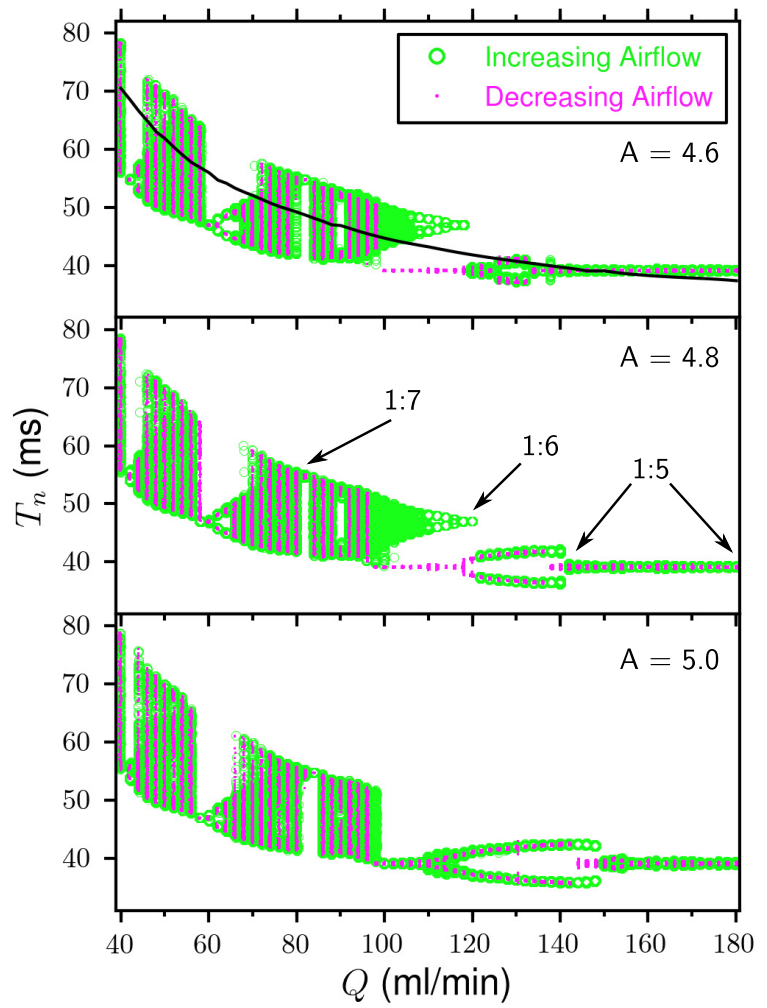

FIG. 4. (Color online) Experimental bifurcation diagrams $T_{n}$ vs $Q$, for $f_{s}=128 \mathrm{~Hz}$ and $H=8.5 \mathrm{~cm}$, obtained by increasing the air flow, in empty light gray circles (green) and decreasing the airflow $Q$, in full dark gray points (magenta) for $A=4.6$ (top panel), $A=4.8$ (middle panel), and $A=5.0$ (bottom panel). The black full line in the top panel represents the bifurcation diagram of a period-1 orbit for no sound amplitude $(A=0)$. Frequency lockings $25.4 / 128=$ $1: 5,21.33 / 128=1: 6$, and $18.28 / 128=1: 7$ are indicated by the arrows.

To construct bifurcation diagrams $T_{n}$ vs $Q$, we increase the air flow in the interval $\left[\begin{array}{ll}40 & 180\end{array}\right] \mathrm{ml} / \mathrm{min}$, in steps of $2 \mathrm{ml} / \mathrm{min}$, having the amplitude of sound $A$ as a fixed parameter. Therefore, we collect data for 71 values of the airflow. We initially set up the air flow in $Q=40 \mathrm{ml} / \mathrm{min}$ and apply no sound wave $(A=0)$. Under these initial conditions, the bubbling presents a stable period-1 behavior. Once that period 1 is achieved, the sound amplifier is turned on at a fixed amplitude, and the data acquisition starts for 71 values of increasing $Q$, until it reaches the maximum value, and another 71 values of decreasing $Q$, until it reaches the minimum value. In Figs. 3 and 4 we show such diagrams $T_{n}$ vs $Q$ with $H=8.5 \mathrm{~cm}$ and $f_{s}=128 \mathrm{~Hz}$, for different values of the sound amplitude $A=4.0,4.2,4.4,4.6,4.8$, and 5.0 (arb. units). Each bifurcation diagram was obtained first increasing the air flow (empty light gray circles - green online) and after decreasing the air flow (full dark gray point-magenta online). Many coexistence of attractors can be identified by the hysteresis. These diagrams show different dynamical behaviors, a variety of bifurcations, crisis, coexistence of attractors, chaotic, and periodic bubbling.

In Fig. 3, for values of $Q$ larger than $Q \approx 115 \mathrm{ml} / \mathrm{min}$, we observe frequency locking $f_{b} / f_{s}=25.4 / 128=1: 5$ related 

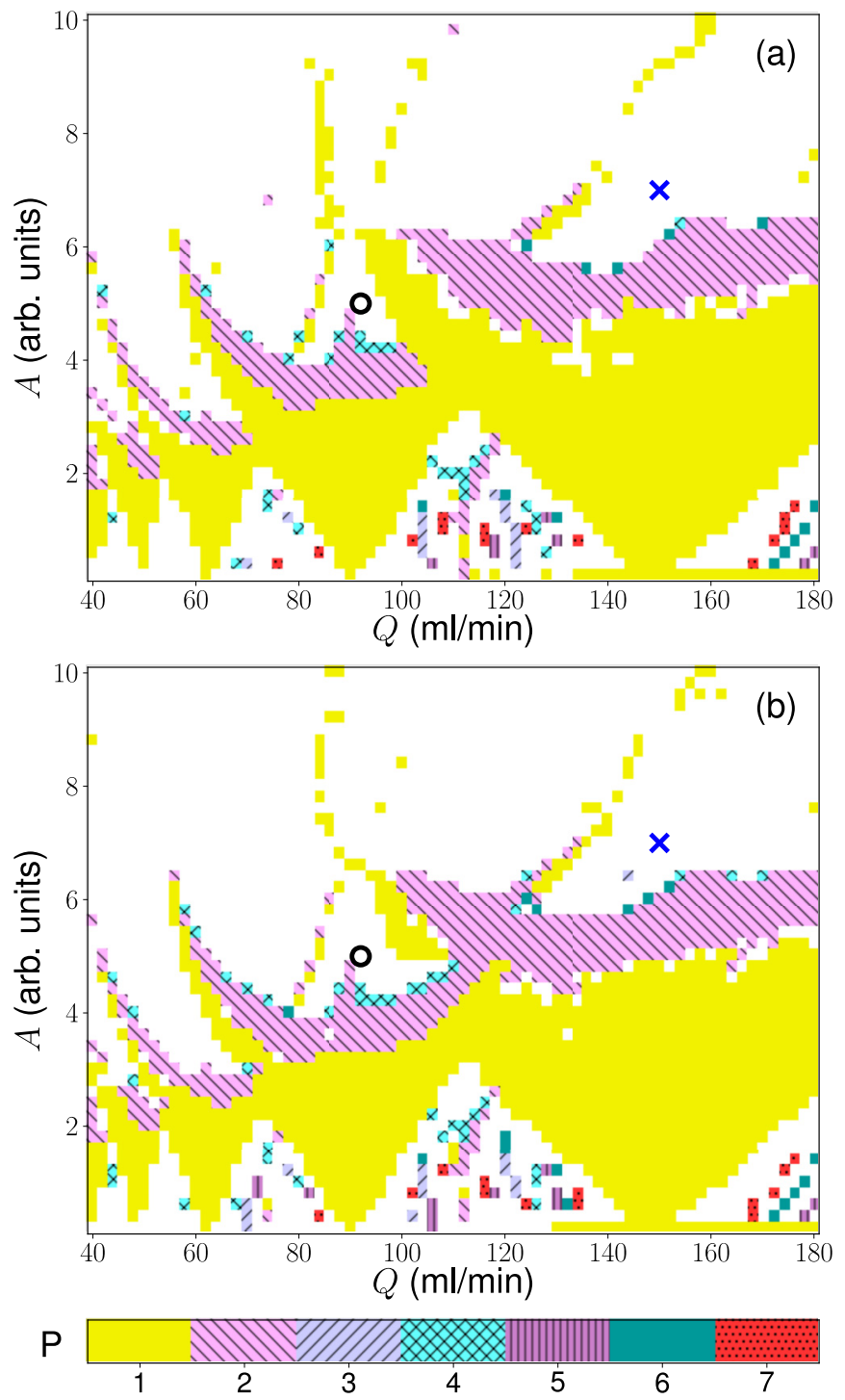

FIG. 5. (Color online) Experimental parameter spaces of periodicity for $H=8.5 \mathrm{~cm}, f_{s}=128 \mathrm{~Hz}$. The different gray level (colored online) regions with different textures show the periodicity $P$ detected. The regions representing parameters of time series with period higher than 7 or not well specified periodicity are shown in white. The black circles correspond to the point $(Q, A)=(92 \mathrm{ml} / \mathrm{min}, 5)$, and the crosses (blue) to the point $(Q, A)=(150 \mathrm{ml} / \mathrm{min}, 7)$.

to a period $P=1$ for all three values of $A$. Frequency lockings $f_{b} / f_{s}=21.33 / 128=1: 6$ are clearly observed in the intervals, [60 70] $\mathrm{ml} / \mathrm{min}$ and [100 115] $\mathrm{ml} / \mathrm{min}$, related to periodic bubbling of periods $P=1$ and $P=2$. For $A=4.2$ and $A=4.4$ frequency lockings $f_{b} / f_{s}=18.28 / 128=1: 7$ related to period $P=1$ around to $Q \approx 80 \mathrm{ml} / \mathrm{min}$ and related to $P=1$ and $P=2$ in the interval [42 47] $\mathrm{ml} / \mathrm{min}$. In Fig. 4 we have similar bifurcation diagrams and frequency lockings, $f_{b} / f_{s}=25.4 / 128=1: 5$ for $P=1$ and $P=2$ periodic behavior cases.

For a global view of the periodic behaviors of this system, we constructed a parameter space $(Q, A)$ of periodicity for $H=8.5 \mathrm{~cm}$ shown in Fig. 5 . We consider 50 values of $A$, and for each value of $A$, we detect the period $P$ of the bubbling for 71 time series, each for a different value of $Q$. The airflow $Q$ is varied in the interval [40 180] $\mathrm{ml} / \mathrm{min}$, by increasing the airflow and after decreasing it, with steps of $2 \mathrm{ml} / \mathrm{min}$, and the sound amplitude is varied in the interval [0.2 10.0], with steps of 0.2 . The attractors periodicity $P$, for every temporal series, was obtained when $\left|T_{n+P}-T_{n}\right| \leqslant 0.2 \mathrm{~ms}$ is satisfied for every $n=1,2,3, \ldots, N$. To every period, from $P=1$ up to $P=7$, we attributed a gray level (color online) and a texture as shown in Fig. 5. Within an Arnold tongue, by increasing the value of $A$, maintaining $Q$ constant, one sees a period doubling bifurcation, where full light gray (yellow online) represents parameters for which $P=1$, slashed dark gray (magenta online) represents parameters for which $P=2$. Periods bigger than 7 or not well defined, as it happens in the case of chaotic bubbling regimes, were plotted with white color. The colored points in the parameter spaces represent parameters for which the time intervals between bubbling is periodic and, in addition, there is frequency locking between the air bubbling and the sound wave, i.e., these areas represent the Arnold tongues of frequency locking. In both parameter spaces shown in Fig. 5, the black empty circles at the point $(Q, A)=(92 \mathrm{ml} / \mathrm{min}, 5)$ and crosses at $(Q, A)=(150 \mathrm{ml} / \mathrm{min}$, 7) are out of any Arnold tongue of frequency locking, meaning that for these parameters the bubbling is neither periodic nor Eq. (4) can be verified. As we shall see in the next section, for these parameters the time intervals for the bubbling is chaotic and there is phase synchronization between the air bubbles and the sound wave.

\section{B. Phase synchronization}

To have a global picture of the types of behaviors as shown in Fig. 5, we have built parameter spaces $(Q, A)$ whose color describes the $p: q$ mode of phase synchronization, defined as in the following. First we need to define the two phases involved. At each launching of a bubble, its phase grows by $2 \pi$, so at the time $t_{k}=\sum_{n=1}^{k} T_{n}$ the phase is given by $\theta_{b}\left(t_{k}\right)=2 \pi k$, while the phase of the sound wave is given by $\theta_{s}\left(t_{k}\right)=2 \pi f_{s} t_{k}$. To measure phase synchronization, we used the generalized phase difference [1]:

$$
\phi_{p, q}(t)=p \theta_{s}(t)-q \theta_{b}(t),
$$

where we considered the smallest $(p, q)$ such that the absolute value of the phase difference is bounded, i.e.,

$$
\left|\phi_{p, q}\right| \leqslant K .
$$

When $\phi_{p, q} \leqslant K$, and $K$ is a finite quantity such that $K \gg$ $\epsilon$, where $\epsilon \approx 2 \times 10^{-3}$, the system is in a $p: q$ phase synchronization. Notice that when a system has quantities that satisfy Eq. (4), they must also satisfy Eq. (7) (see Ref. [41]). In other words, if a system presents frequency locking [so the motion is periodic and Eq. (4) is satisfied], these quantities must also satisfy Eq. (7), and therefore the system presents phase synchronization and Eq. (7) is satisfied. On the other hand, satisfying Eq. (7) does not guarantee the existence of frequency locking, since the existence of a periodic motion cannot be stated, unless $\left|\phi_{p, q}\right|=0$.

In Fig. 6 we show experimental parameter spaces of phase synchronization, where we show by color code and different textures the values of $p$ and $q$ that not only minimize $\phi_{p, q}$ but 

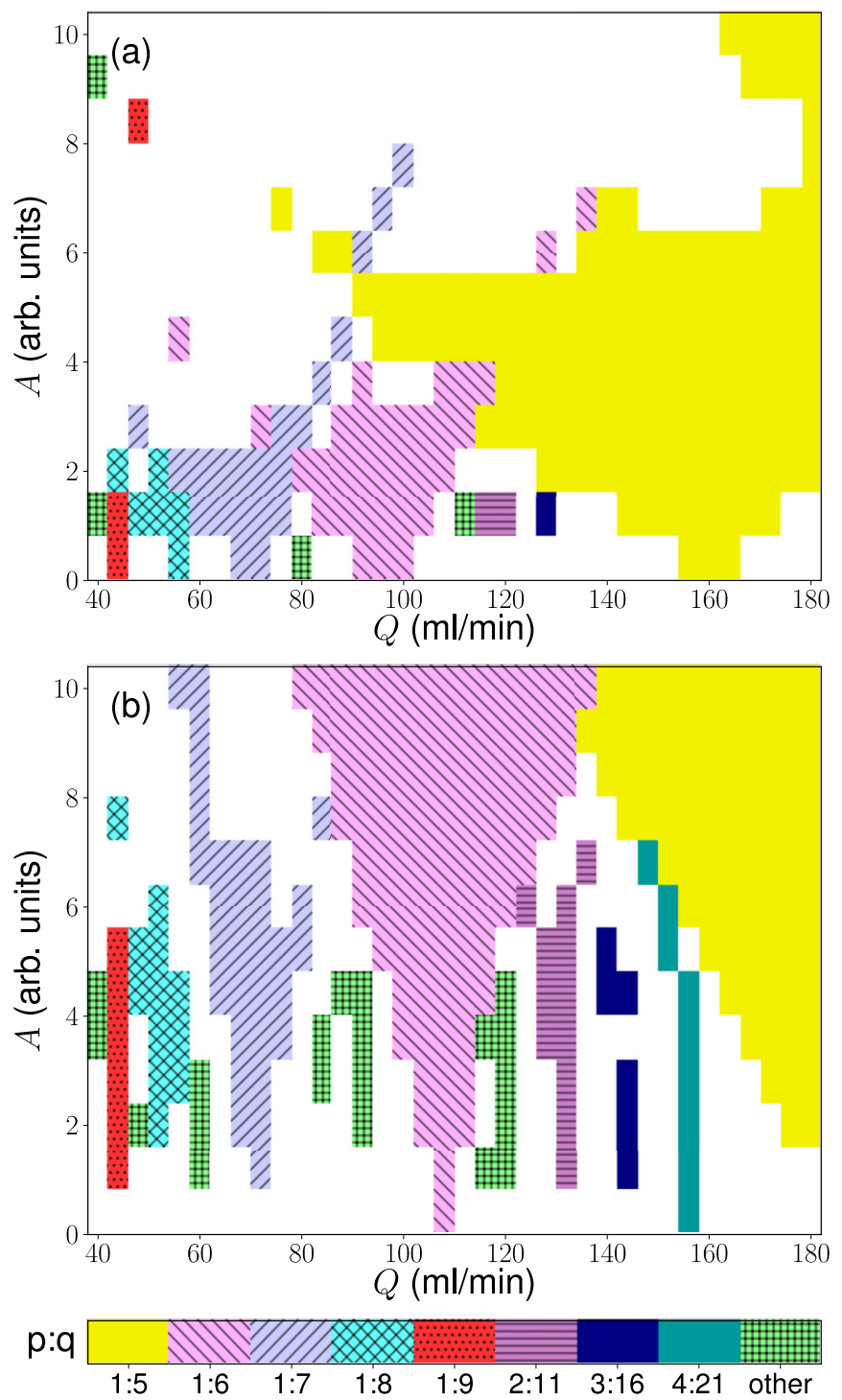

FIG. 6. (Color online) Experimental parameter space of synchronization: (a) $H=4.0 \mathrm{~cm}, f_{s}=120 \mathrm{~Hz}$, (b) $H=13.0 \mathrm{~cm}, f_{s}=$ $139 \mathrm{~Hz}$.

also satisfy inequality in (7). The two $H$ values considered, $H=4 \mathrm{~cm}$ and $H=13 \mathrm{~cm}$, correspond to the lower and higher bounding values within which we can obtain data with a small signal/noise relation. The Arnold tongues of phase synchronization [defined by Eq. (7)] are represented by colors and texture, showing a strong dependence on $H$.

With the same data of Fig. 5 for $H=8.5 \mathrm{~cm}$, the corresponding parameter spaces of phase synchronization obtained with Eqs. (6) and (7) are shown in Fig. 7. Now we can see that the empty black circle $(Q, A)=(92 \mathrm{ml} / \mathrm{min}$, 5) and cross $(Q, A)=(150 \mathrm{ml} / \mathrm{min}, 7)$ are inside of Arnold tongues of phase synchronization. By comparison with the respective parameter space of periodicity in Fig. 5, we conclude that for these points we are observing an aperiodic oscillation, an indication of chaotic phase synchronization. To confirm this assumption, we construct the first return maps of these two time series shown in Fig. 8(a). These maps have a topology (see Ref. [33,34]) that provides all
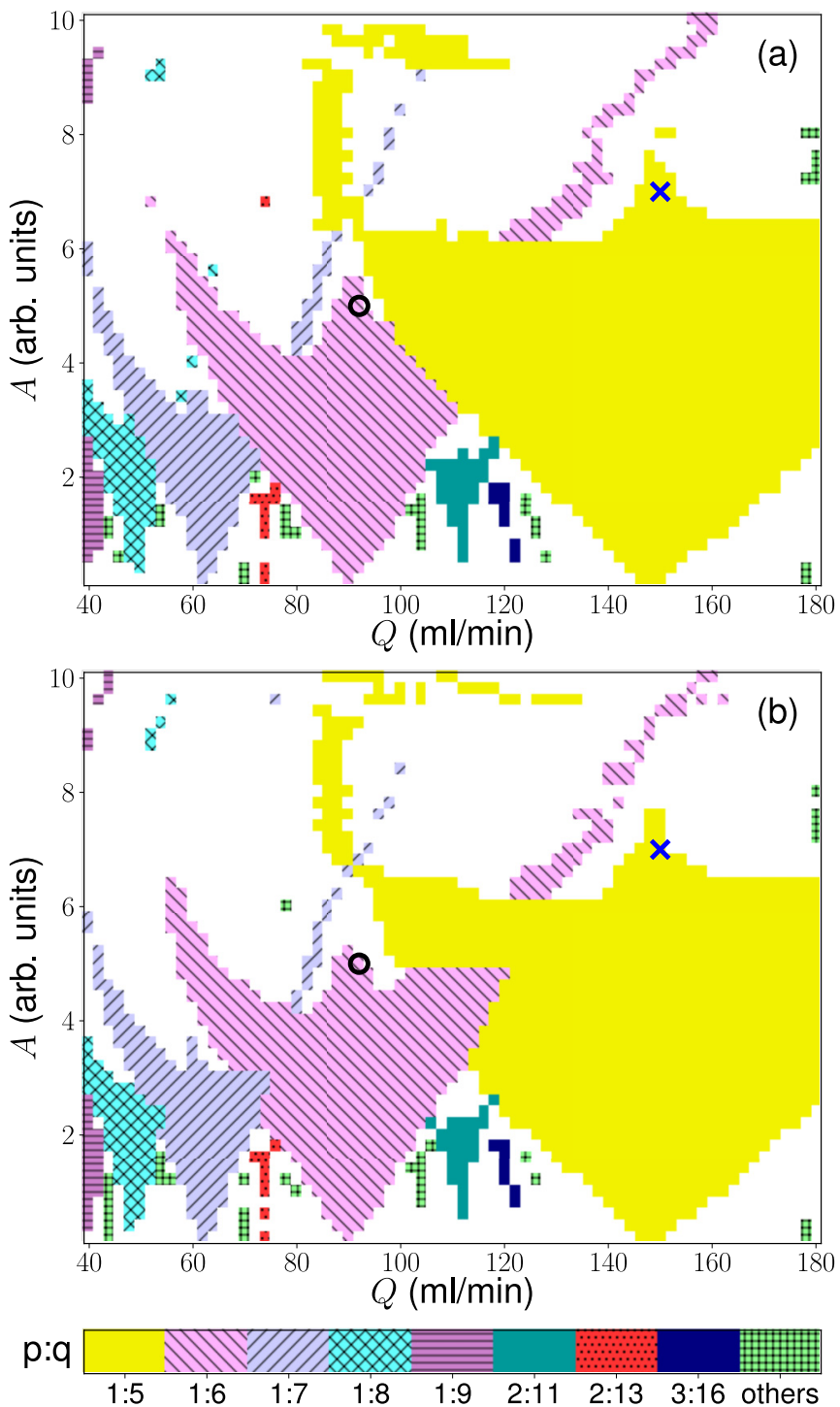

FIG. 7. (Color online) Experimental parameter spaces of synchronization for $H=8.5 \mathrm{~cm}$ and $f_{s}=128 \mathrm{~Hz}$. The black empty circles correspond to the point $(Q, A)=(92 \mathrm{ml} / \mathrm{min}, 5)$, and the crosses (blue) to the point $(Q, A)=(150 \mathrm{ml} / \mathrm{min}, 7)$. (a) Increasing the airflow and (b) decreasing the airflow. It is possible to see a sequence of Arnold tongues appearing with a ratio $p / q$ respecting a Farey tree, where the Arnold tongue 2:11 appears in between two Arnold tongues, one with $1: 5$ and another with $1: 6$, and the Arnold tongue $3: 16$ appears in between one with $2: 11$ and another with $1: 5$. Also the Arnold tongue $2: 13$ appears in between one with $1: 7$ and another with $1: 6$.

conditions for chaos: unstable periodic orbits, transitivity, mixing, and sensibility to initial conditions. For the case of $(Q, A)=(92 \mathrm{ml} / \mathrm{min}, 5)$ (black circles) the experimental bubbling frequency is $f_{b}=21.34$ bubbles/s since $f_{s}=128$ $\mathrm{Hz}$, then $f_{s} / f_{b}=6$. For $(Q, A)=(150 \mathrm{ml} / \mathrm{min}, 7)$, in light gray (green online) the experimental bubbling frequency is $f_{b}=25.595$ bubbles $/ \mathrm{s}$ then $f_{s} / f_{b}=5$, implying $1: 6$ and $1: 5$ chaotic phase synchronizations. Another evidence that these are indeed cases of chaotic phase synchronization (and not of frequency locking), in addition to verifying Eq. (7), is 


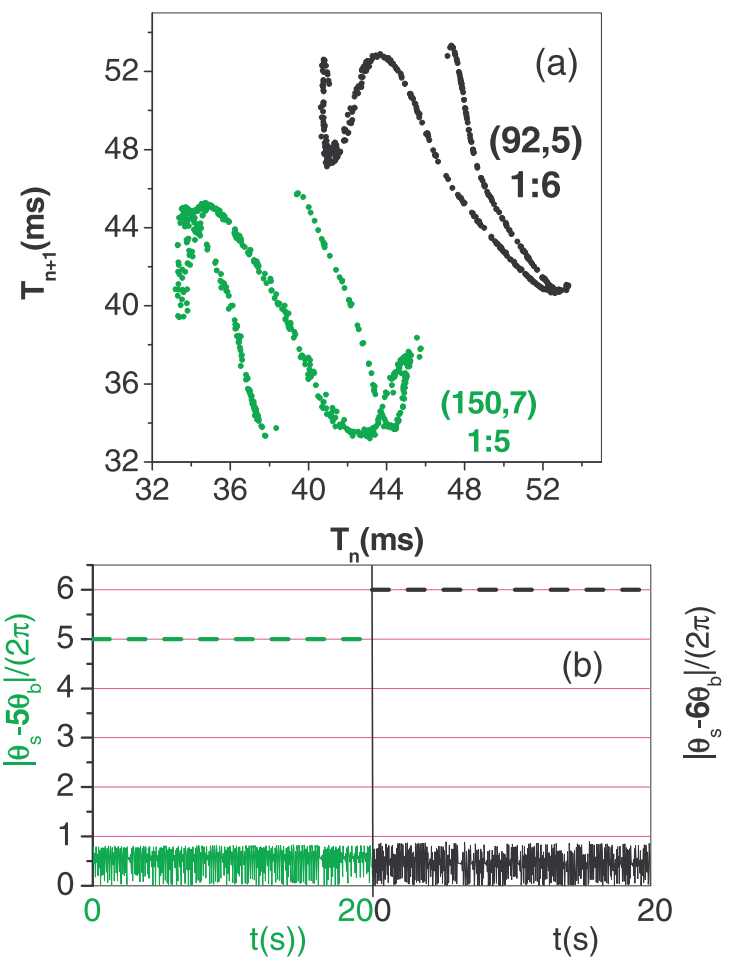

FIG. 8. (Color online) $H=8.5 \mathrm{~cm}$ and $f_{s}=128 \mathrm{~Hz}$, in (a) the chaotic first return maps $T_{n+1}$ vs $T_{n}$. Black dots are obtained for $(Q, A)=(92 \mathrm{ml} / \mathrm{min}, 5)$ and $f_{b}=21.34$ bubbles $/ \mathrm{s}$. Gray dots (green) are obtained for $(Q, A)=(150 \mathrm{ml} / \mathrm{min}, 7)$ and $f_{b}=25.595$ bubbles/s. In (b) the plots of phase difference $\left|\phi_{p, q}(t)\right|$ side by side corresponding to chaotic synchronizations $1: 5$ in gray (green) and $1: 6$ in black. The dashed horizontal lines correspond to the bound $\max (p, q) /(2 \pi)$ for synchronization.

that $\phi_{p, q}(t)>\epsilon$ as shown in Fig. 8(b). Therefore, a periodic attractor within the region of the frequency locking Arnold tongue bifurcates into a chaotic one producing chaotic phase synchronization.

\section{MODELING}

We adapted a model presented in Ref. [38] to simulate the effect of the sound wave signal into the bubble dynamics.

We consider a single bubble being formed with a volume $V(z)$, with $z(t)$ being the bubble size in the vertical direction, measured from the top of the nozzle as shown in a zoomed view in Fig. 1:

$$
\begin{aligned}
\dot{\mathfrak{p}} & =\frac{1}{V_{o}}[R T Q-\mathfrak{p} \dot{V}(z)], \\
\ddot{z} & =\frac{1}{a(z)}\left|\left[\mathfrak{p}-\mathfrak{p}_{e}(z)\right]-b(z) \dot{z}-c(z) \dot{z}^{2}\right|,
\end{aligned}
$$

where $R, T$, and $V_{o}=V_{M}+V_{L}=14.2 \mathrm{ml}$ are constants; and $V(z), a(z), b(z)$, and $c(z)$ are functions related with the flow regime and depend on the geometrical parameters $h, L, V_{o}$, and $r_{0}$ of the system, see the Appendix of Ref. [38] for details. The dynamic variables are the pressure inside the syringe chamber $\mathfrak{p}, z$, and $\dot{z} \cdot \mathfrak{p}_{e}(z)$ is the equilibrium pressure, which is the sum of the hydrostatic pressure and the surface tension in the interface air/solution. The instants of the bubble detachments are given by an equilibrium formula when the upward force (buoyancy) is equal to the downward forces (surface tension and viscous drag). The interaction between the bubble and the sound wave was modeled by perturbing the equilibrium pressure $\mathfrak{p}_{e}$ with a resonant sound wave:

$$
\mathfrak{p}_{e}^{s}=\mathfrak{p}_{e}+A_{s} \cos \left(2 \pi f_{s} t\right),
$$

where $A_{s}$ and $f_{s}=128 \mathrm{~Hz}$ are, respectively, the amplitude and the frequency of the perturbation. To detect synchronization between the perturbing wave and the bubbles, we consider time series numerically obtained for the instants of detachment of the bubbles.

The parameter space of phase synchronization is shown in Fig. 9, obtained by verifying Eq. (7), for the lowest values
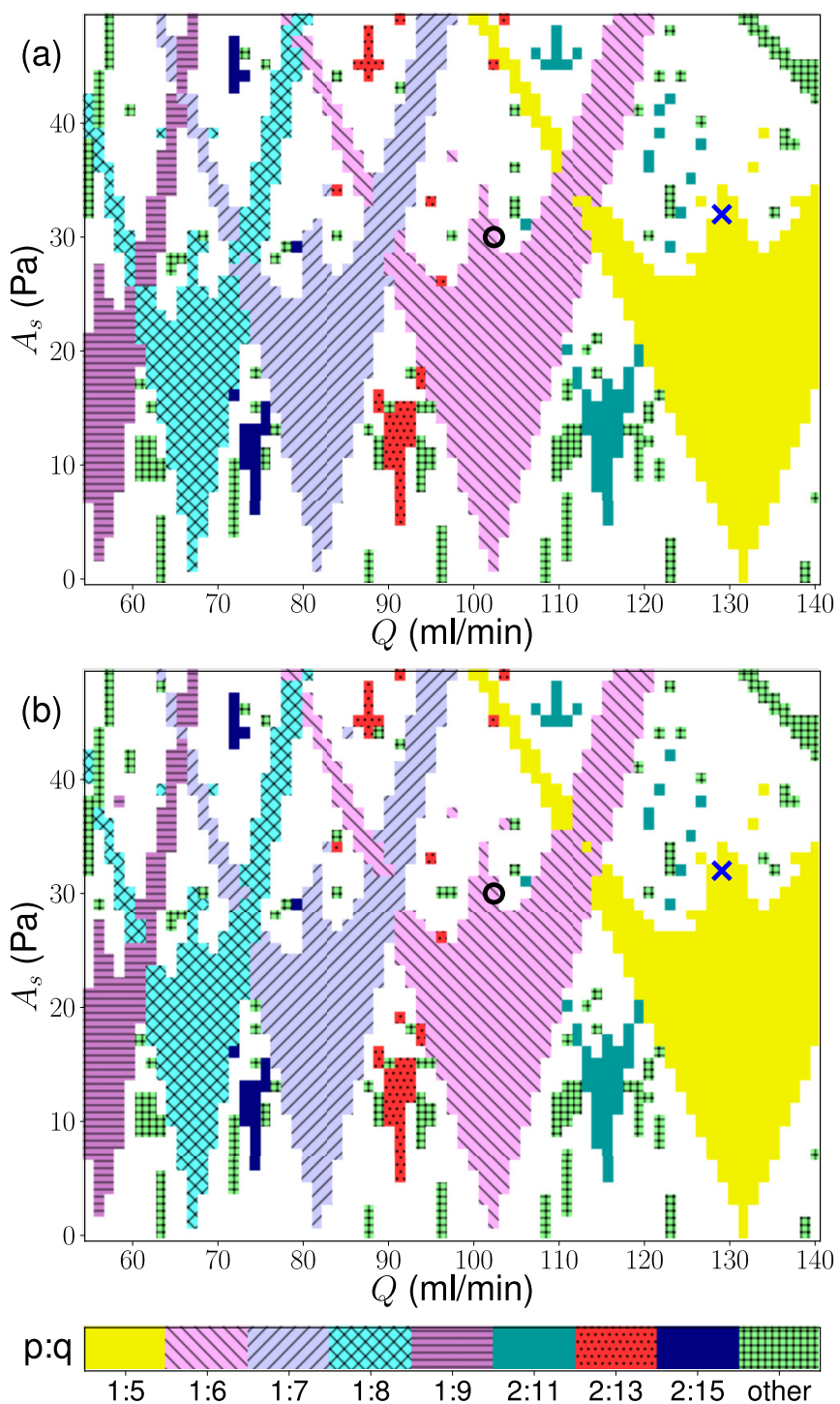

FIG. 9. (Color online) Simulated parameter space of phase synchronization [Eq. (7)] $f_{s}=128 \mathrm{~Hz}$. The black empty circles are the point $\left(Q, A_{s}\right)=(102.4 \mathrm{ml} / \mathrm{min}, 30 \mathrm{~Pa})$, and the crosses (blue) correspond to $\left(Q, A_{s}\right)=(129 \mathrm{ml} / \mathrm{min}, 32 \mathrm{~Pa})$, whose time series are examples of chaotic phase synchronization. Sequences of Arnold tongues respecting the Farey tree can also be observed, as observed experimentally. 


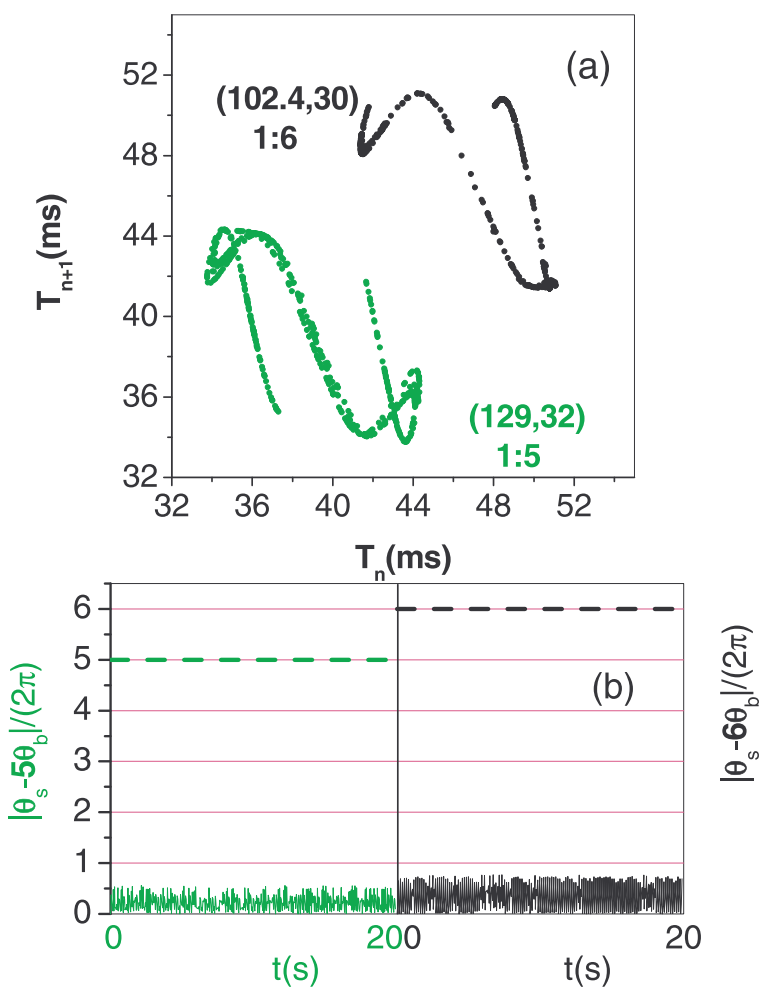

FIG. 10. (Color online) For $f_{s}=128 \mathrm{~Hz}$. In (a) the simulated chaotic attractors for $(Q, A)=(102.4 \mathrm{ml} / \mathrm{min}, 30 \mathrm{~Pa}), f_{b}=21.333$ bubbles/s in black dots; and for $(Q, A)=(129 \mathrm{ml} / \mathrm{min}, 30 \mathrm{~Pa})$, $f_{b}=25.60$ bubbles $/ \mathrm{s}$ in gray dots (green). In (b) the plots of phase difference $\left|\phi_{p, q}(t)\right|$ side by side corresponding to chaotic synchronizations $1: 5$ in gray (green) and $1: 6$ in black. The dashed horizontal lines correspond to the bound $\max (p, q) /(2 \pi)$ for synchronization.

of $p$ and $q$, by decreasing (a) and increasing (b) the airflow. Notice that we observe the same $p, q$ Arnold tongues obtained experimentally $(p: q=1: 5,1: 6,1: 7,1: 8,1: 9,2: 11,2$ : 13). In this numerical simulations, the higher-order Arnold tongues, appearing in between the main ones (forming a Farey tree), are better recognized. The black and the light gray points correspond to the points $\left(Q, A_{s}\right)=(102.4 \mathrm{ml} / \mathrm{min}$, $30 \mathrm{~Pa})$ and $\left(Q, A_{s}\right)=(129 \mathrm{ml} / \mathrm{min}, 32 \mathrm{~Pa})$, respectively, where we observe chaotic phase synchronization with the chaotic attractors similar to the experimental ones, as shown in Fig. 10(a). The positiveness of $\left|\phi_{p, q}\right|$, another evidence for chaotic phase synchronization, is shown in Fig. 10(b).

\section{CONCLUSIONS}

We showed experimentally that air bubbles expelled from a nozzle immersed in a viscous fluid can be both frequency locked and phase synchronized with the external sound wave. We observed coexistence of attractors through hysteresis by increasing the airflow $Q$ followed by a decreasing of it. Such complex behavior and many modes of synchronization were shown to depend on the air flows, the height $(H)$ of the solution above the top of the nozzle, and the amplitude of the sound wave. Chaotic phase synchronization appears in the top region of the Arnold tongues that describe periodic frequency locking. Therefore, a periodic attractor bifurcates into a chaotic one producing phase synchronization. The experimental results were reproduced by numerical simulations of a single model for a bubble growing that interacts with a fluid whose movement is being disturbed by a sound wave, a phenomenon that was modeled by changing the equilibrium pressure parametrically with a cosine wave. This model, despite being very simple, can capture the main characteristics of the dynamics of formation of air bubbles under an external resonant perturbation.

\section{ACKNOWLEDGMENTS}

We thank the São Paulo State Agency FAPESP and the Federal Brazilian Agency $\mathrm{CNPq}$ for the financial support. M.S.B. acknowledges EPSRC Grant No. EP/IO32606/1.
[1] A. Pikovsky, M. Rosenblum, and J. Kurths, Synchronization: A Universal Concept in Nonlinear Sciences (Cambridge University Press, Cambridge, 2001).

[2] L. Glass, Nature (London) 410, 277 (2001).

[3] B. Blasius, A. Huppert, and L. Stone, Nature (London) 399, 354 (1999).

[4] M. Rosenblum, G. Kuuz, and B. Pompe, in Nonlinear Analysis of Physiological Data, edited by H. Kantz, J. Kurths, and G. Mayer-Kress (Springer, Berlin, 1998), pp. 283-306.

[5] C. Schäfer, M. G. Rosenblum, H. H. Abel, and J. Kurths, Phys. Rev. E 60, 857 (1999).

[6] D. Terman, N. Kopell, and A. Bose, Physica D 117, 241 (1998).

[7] A. Bose, N. Kopell, and D. Terman, Physica D 140, 69 (2000).

[8] C. C. Chow, Physica D 118, 343 (1998).

[9] A. Arenas, Albert Daz-Guilera, J. Kurths, Y. Morenob, and C. Zhoug, Phys. Rep. 424, 175 (2006).
[10] S. Boccaletti, J. Kurths, G. Osipov, D. L. Valladares, and C. S. Zhou, Phys. Rep. 366, 1 (2002).

[11] A. A. Kulkarni and J. B. Joshi, Ind. Eng. Chem. Res. 44, 5873 (2005).

[12] S. Xie and R. B. H. Tan, Chem. Eng. Sci. 58, 4639 (2003).

[13] M. Ruzicka, J. Drahos, J. Zahradník, and N. H. Thomas, Chem. Eng. Sci. 55, 421 (2000).

[14] F. A. C. Pereira, E. Colli, and J. C. Sartorelli, Phys. Rev. E 87, 022917 (2013).

[15] P. Snabre and F. Magnifotcham, Eur. Phys. J. B 4, 369 (1998).

[16] Equilibrium pressure is defined as the sum of the hydrostatic pressure and the surface tension in the interface air/solution.

[17] M. S. Baptista, C. Zhou, and J. Kurths, Chin. Phys. Lett. 23, 560 (2006).

[18] I. Siddiqi, R. Vijay, F. Pierre et al., Phys. Rev. Lett. 94, 027005 (2005)

[19] M. S. Baptista and I. L. Caldas, Phys. Rev. E 58, 4413 (1998). 
[20] M. S. Baptista and I. L. Caldas, Nonlinear Dyn. 17, 119 (1998).

[21] Arnold tongues are parameter regions that represent periodic motion. In Ref. [22] Arnold wanted to understand how a small periodic perturbation can turn a quasiperiodic oscillation in a periodic one. He studied the circle map perturbed by a sinusoidal term $\left[\theta_{n+1}=\theta_{n}+\omega+K / 2 \pi \sin \left(2 \pi \theta_{n}\right)\right]$. The circle map for $K=0$ has Lebesgue measure one of presenting quasiperiodic motion. As the perturbation is introduced $(K \neq 0)$, there can exist periodic motion. The larger the value of $K$, the larger the interval of the natural frequency $\omega$ that produces periodic motion. A nice tutorial over the topic can be seen in the book [23].

[22] V. I. Arnold, Amer. Math. Soc. Trans. 46, 213 (1965).

[23] J. H. Argyris, G. Faust, and M. Haase, An Exploration of Chaos (Elsevier, Amsterdam, 1994).

[24] M. P. Kennedy, H. Mo, and X. Dong, in 20th European Conference on Circuit Theory and Design (ECCTD Linkoping, Sweden, 2011).

[25] When a chaotic oscillator is periodically perturbed, the process by which chaotic phase synchronization appears is similar to the process by which periodic motion appears in the perturbed circle map [26]. The parameter regions that represent parameters that produce chaotic phase synchronization are also denoted by Arnold tongues.

[26] M. G. Rosenblum, A. S. Pikovsky, and J. Kurths, Phys. Rev. Lett. 76, 1804 (1996).

[27] M. S. Baptista, T. P. Silva, J. C. Sartorelli, I. L. Caldas, and E. Rosa, Jr., Phys. Rev. E 67, 056212 (2003).

[28] A. Uchida, R. McAllister, and R. Roy, Phys. Rev. Lett. 93, 244102 (2004).
[29] K. A. Mirus and J. C. Sprott, Phys. Rev. E 59, 5313 (1999).

[30] R. Chacon, Phys. Rev. Lett. 86, 1737 (2001).

[31] A. Kandangath, S. Krishnamoorthy, Y.-C. Lai, and J. A. Gaudet, IEEE Trans. Circ. Syst. I 54, 1109 (2007).

[32] Y.-C. Lai, A. Kandangath, S. Krishnamoorthy, J. A. Gaudet, and A. P. S. de Moura, Phys. Rev. Lett. 94, 214101 (2005).

[33] A. Tufaile and J. C. Sartorelli, Phys. Lett. A 275, 209 (2000).

[34] A. Tufaile and J. C. Sartorelli, Physica A 275, 336 (2000).

[35] A. Tufaile and J. C. Sartorelli, Phys. Lett. A 287, 74 (2001).

[36] V. S. M. Piassi, E. Colli, A. Tufaile, and J. C. Sartorelli, Chaos Solitons Fractals 41, 1041 (2009).

[37] J. Hengtai, K.-T. Tsai, and L.-W. Kuo, Chaos 23, 033126 (2013).

[38] F. A. C. Pereira, E. Colli, and J. C. Sartorelli, Chaos 22, 013135 (2012).

[39] J. C. Sartorelli, W. M. Gonçalves, and R. D. Pinto, Phys. Rev. E 49, 3963 (1994).

[40] See Supplemental Material at http://link.aps.org/supplemental/ 10.1103/PhysRevE.90.042902 for the movie which was taped at 500 frames/s and shown at 30 frames/s for $Q=126 \mathrm{ml} / \mathrm{min}$. Initially, the video shows a $P=1$ periodic bubbling when no external sound wave $(A=0)$ is applied. Then, the external sound wave was turned on $(A=5)$, and the bubbling evolution to a periodic $P=2$ behavior can be followed.

[41] Departing from Eq. (4), we can write that $f_{s} p-f_{b} q=0$, which can be written as $f_{s} p-q / T_{b}=0$. Multiplying this equation by $2 \pi$, and using that for large $k, T_{b}=t_{k} / k$, we obtain that $2 \pi f_{s} p-2 \pi q k / t_{k}=0$. Multiplying this equation by $t_{k}$, we have that $2 \pi t_{k} f_{s} p-2 \pi q k=0$. Since $\theta_{s}=2 \pi f_{s} t_{k}$ and $\theta_{b}=2 \pi k$, we arrive that $p \theta_{s}-q \theta_{b}=0$. Therefore, if Eq. (4) is satisfied, then Eq. (7) must also be satisfied. 